\title{
CONFERENCE SUMMARY: GLIMPSE OF A NEW FUTURE
}

\author{
S. M. FABER \\ UCO/Lick Observatory, \\ University of California, Santa Cruz, CA 95064
}

\begin{abstract}
Research on galaxy formation is about to be inundated with data from the distant Universe and from highly realistic model simulations. First glimpses from this and other work are already showing the need for new paradigms of galaxy formation.
\end{abstract}

\section{Introduction}

Some conferences are remembered as landmark events where consensus on important new paradigms is achieved. This conference in contrast may be remembered as the first glimpse of a new future for galaxy evolution studies - an era dominated by incredibly realistic simulations and data from the distant Universe. The need for traditional, local data will not diminish - to the contrary, the new techniques will stimulate comprehensive surveys of the local Universe as never before. At this conference we saw the first trickle of such new results - the first drops splattering down from the bursting dam above. Shortly a flood of new results will be upon us, carrying us in its wake. Such floods have a tendency to scour the landscape, and this one promises to be no different. Heidelberg may be the last time we stood and viewed the forest as it once seemed to be.

\section{Interactions and Mergers}

A major topic at this conference was galaxy interactions. Freeman, Sancisi, and Kennicutt all argued for continuous merging of small satellite galaxies up through recent times. We badly need to describe this process quantitatively: what fraction of the baryonic mass of a normal field spiral like the Milky Way arrived how recently, and in what-sized pieces? Although the quantitative impact is still not clear, incontrovertible evidence such as kinematic subsystems and tidally stripped satellites of the Milky Way 
establish that at least some material arrived in this form. An implication is that an object like the Galactic halo, built up via many accretions over time, may not be describable by simple two-integral dynamical models.

Barnes emphasized that merger remnants can retain detailed memory of the kinematics of the individual progenitors. Just a few percent of gas appears to have a profound regularizing effect on the dynamical structure of the remnant by erasing box orbits and reducing triaxiality. Triaxial galaxies such as certain massive ellipticals must evidently have formed in mergers that were nearly gas-free.

Important new light was shed on dynamical interactions of galaxies in clusters. Moore argued that galaxies in clusters are subject to repeated highvelocity bombardment at speeds in excess of the internal escape velocity. A fascinating video by Moore and collaborators illustrated this "harassment," showing outer disks being whittled away and perturbed gas falling to the galaxy centers. Both tendencies would be helpful in explaining cluster S0's. In the conference's best one-two punch, this talk was followed by Sharpless' description of an S0-dominated sub-cloud in Coma. The high Balmer strengths in this cloud may have resulted from harassment-triggered starbursts as the cloud amalgamated with the main body of the cluster.

\section{Dynamics of Spheroids and Bulges}

"Mass easily finds the center" was an important theme of several talks. Burkert emphasized a puzzle with hierarchical clustering, which tends to produce central cusps of dark matter even without gasesous dissipation. Gas exacerbates the problem, as illustrated in videos of merging, forming, and harassed galaxies by Barnes, Steinmetz, and Moore. Disturbed gas in the outer parts falls unerringly to the center in just a few timescales. Why, then, aren't the centers of galaxies denser than they are? Does the gas supply simply run out, or does feedback from star formation or AGNs somehow truncate further infall?

It was once possible to regard the centers of galaxies as separate entities with a distinct evolutionary history from the main body. That view is less tenable now as we see that dense central structures survive mergers and thus in some sense preserve the formation history of the whole galaxy. The elliptical core parameter relations, described by Kormendy based on new HST images, are thus doubly puzzling - what process impressed a core length-scale separate from the global radius, and why aren't these cores of the classic type (as in the isothermal sphere) but rather shallow power laws? The Universe, surprisingly, does not seem to make classic cores in galaxies.

This conference saw a landmark paper (Jeske et al.), which broke the 
longstanding degeneracy on elliptical galaxy mass measurements. A wide variety of mass profiles are consistent with given photometric and dispersion profiles provided the radial velocity-dispersion anisotropy is adjusted. Jeske et al. added kurtosis, $h_{4}$, and obtained, for the first time, clear evidence of dark matter at about $R_{e}=1$. The new technique opens the way to mass profiles of ellipticals with simple orbital structure. Similarly, Scorza showed how $h_{4}$ plus $h_{3}$ (skewness) can be used to decompose disky ellipticals and S0's into disk and spheroid components. Line profile shape is clearly developing into the key dynamical tool that many hoped it would be.

De Zeeuw discussed new work linking HST core mass profiles with global galaxy dynamics. Merrit and Fridman (preprint) have shown that stars in box orbits (as in triaxial galaxies) will eventually be scattered by a steep central density cusp. This means that small ellipticals, which always show steep central cusps (Kormendy) cannot be triaxial, in keeping with their high rotation and disky isophotes. The clear triaxiality of certain massive ellipticals may be slowly dissolving with time as the galaxies evolve toward spherical symmetry. Potentially this could be used to date interactions.

Major new results on bars and bulges were presented. Several speakers suggested that bars may be cyclical, forming from disk instabilities and dissolving slowly to form bulges. There was no clear consensus on whether the Galactic bulge was part of our newly discovered bar, the inner puffed-up disk, or simply the inner part of the metal-poor spheroid. Peletier stressed that bulges are more similar in color to their associated disks than they are to one another, consistent with formation from the same disks. Perhaps there are two kinds of bulges: the classic type related to elliptical spheroids (as in the Sombrero) and smaller bulges in later-type spirals formed from disks.

Lest anyone hope that spheroid dynamics is getting simpler, Bender reminded us of the important elliptical NGC 4365, which shows two kinematically decoupled stellar substreams in its inner spectra yet at most only small departures from regular isophotes. A detailed formation scenario for this Rosetta-Stone object is badly needed.

\section{Stellar Populations}

Age-dating of old stellar populations was the subject of considerable controversy. Davies and Worthey reviewed $\mathrm{H} \beta$ evidence that light-weighted mean ages in some ellipticals, particularly small ones, are as low as a few Gyr. According to this interpretation, the sequence of colors and spectral line strength along the Fundamental Plane owes as much to age differences as to metallicity. An objection is that the Fundamental Plane should be underpopulated at low luminosities in distant clusters, which has so far not 
been observed. The narrowness of the FP locally is also problematic if there is a large age spread at each location. Chiosi presented UV spectra based on models of the hot horizontal branch and showed that $(1550-V)$ color is helpful in distinguishing young single-burst populations from mixtures of old and young stars. On that basis he suggested that M32's stars are a mix of ages rather than exclusively young.

Worthey reminded us of how serious current model uncertainties are in light of the strongly non-solar element ratios that are found in massive ellipticals. Since model spectra are even more sensitive to $Z$ than to age, it may be critical to model these ratios faithfully to obtain reliable absolute calibrations from models.

Bruzual described recent intercomparisons and tests of population models by Bruzual-Charlot, Worthey, and Bressan et al. Disagreements are present - at typically the $25 \%$ level in $M / L$ and age - but the progress made in the last 5 years is significant. All zeropoints are questionable, but relative trends vs. age and $Z$ are now well established. The next challenge is to model the element ratios, and until that is explored, all fitted ages and other absolute quantitities should be regarded as uncertain.

\section{Distant Galaxies}

Data on distant galaxies were to me the most novel and exciting aspect of the conference. Definitive results of the landmark Canada-France redshift survey (Lilly) are now emerging, several talks and posters described morphological results from HST, and the first results on Keck redshifts were presented.

A major breakthrough seems to be some level of agreement on the long-running faint-blue-galaxy problem. Several groups now concur (Lilly, Driver, Marzke) that the excess of faint blue galaxies is due to two effects: more such galaxies locally than previously known, plus a modest increase in either the number density or luminosities of such galaxies in the past. This apparent evolution in the tail of the Hubble sequence seems to be counterbalanced by relative stability in the luminosity function (Lilly) and linewidth properties (Rix, Franx) of early types. Examples of normallooking ellipticals and spirals have been identified at redshifts as early as 0.8 (Koo).

Several speakers discussed morphologies of faint galaxies from HST. Oemler echoed the thoughts of many in noting that distant galaxies look distinctly different from local Hubble types - balanced, symmetric spirals are much rarer - but that some vague correspondence with standard Hubble types seems to be retained. A speculation is that we are seeing back to a time when the foundations of the present Hubble sequence were 
being laid, which opens all sorts of new avenues for exciting research. Interestingly, the morphological peculiarities of field and cluster galaxies look hauntingly similar despite their different environments. A more quantitative characterization of "peculiar" is clearly needed, however.

In one of the rare theoretical papers of the conference, Kauffmann described her program to model galaxy formation schematically using Press-Schechter clustering statistics for the merging hierarchy. These are toy models with many assumptions, but they do allow one to explore quickly the consequences of varying assumptions about star formation, gaseous infall, and other variables. They account quite nicely for the morphologydensity relation and predict, among other things, that stars in ellipticals formed over a wide range of epochs during repeated bouts of merging. An interesting prediction worth testing is that small satellites are accreted constantly onto $L^{*}$ galaxies, reminiscent of the evidence from local galaxies mentioned in Section 1.

I close with a rampant speculation that is not well grounded but might be able to organize much of the observational data on both local and distant galaxies reported at this conference. The idea is that the Hubble sequence is really two distinct phenomena. The first part consists of the classic Hubble types at the head of the sequence ending at about Sc (where Hubble originally truncated the sequence). These bright galaxies all have about the same average luminosity, mass, and surface brightness, show tight structural scaling relations, and are evidently rather stable in properties and number densities out to early epochs. The second half is the irregular tail of the sequence, which shows a wider range of luminosities and surface brightnesses, a distinct Hubble type-luminosity correlation, and is apparently strongly evolving over recent epochs. Talks by Kennicutt, Sancisi, and McGaugh listed other properties that seem consistent with this crude dichotomy. Perhaps the explanation is simply that the first class formed from strong density perturbations early in the Universe, while the second class consists of latecomers whose formation dribbles on to the present. Clearly, the coming flood of realistic simulations and distant survey data will be crucial in answering this and other key questions of galaxy formation. 